\title{
Web of Science
}

1) Clarivate Analytics
Search Search Results

Full Text from Publisher
Tools $\vee$ Searches and alerts $~$

Search History

Marked List

Save to EndNote online

Add to Marked List
In situ transesterification of solid coconut waste in a packed bed reactor with CaO/PVA catalyst

By: Talha, NS (Talha, Nur Syakirah) ${ }^{[1]} ;$ Sulaiman, S (Sulaiman, Sarina) ${ }^{[1]}$

\section{WASTE MANAGEMENT}

Volume: 78 Pages: 929-937

DOI: 10.1016/j.wasman.2018.07.015

Published: AUG 2018

Document Type: Article

View Journal Impact

\section{Abstract}

In this study, solid coconut waste and CaO/PVA was used as raw material and catalyst respectively to produce biodiesel through in situ transesterification. Both, raw material and catalyst were packed in a packed bed reactor. The reaction was fixed for $3 \mathrm{~h}$ and the mixing was kept constant at $350 \mathrm{rpm}$. The highest biodiesel yield of $95 \%$ was obtained at reaction temperature of 61 degrees $\mathrm{C}$ with catalyst loading (CaO/PVA) of $2.29 \mathrm{wt} \%$ and methanol to solid ratio of 12:1. CaO-waste derived catalyst has been successfully proven to be utilized as heterogeneous base catalyst for the production of biodiesel from solid coconut waste. (C) 2018 Elsevier Ltd. All rights reserved.

\section{Keywords}

Author Keywords: Waste-derived catalyst; Biodiesel; In situ transesterification; Polyvinyl alcohol; Eggshell; Solid coconut waste

KeyWords Plus: BIODIESEL PRODUCTION; SUNFLOWER OIL; SOYBEAN OIL; PALM OIL; SHELL; OPTIMIZATION; EGGSHELL; EXTRACTION; RAPESEED; KINETICS

\section{Author Information}

Reprint Address: Sulaiman, S (reprint author)

+ Int Islamic Univ Malaysia, Dept Biotechnol Engn, Kulliyyah Engn, POB 10, Kuala Lumpur 50728, Malaysia. Addresses:

+ [1] Int Islamic Univ Malaysia, Dept Biotechnol Engn, Kulliyyah Engn, POB 10, Kuala Lumpur 50728, Malaysia E-mail Addresses: sarina@iium.edu.my

\section{Funding}

\begin{tabular}{|l|l|}
\hline Funding Agency & Grant Number \\
\hline $\begin{array}{l}\text { Ministry of Higher Education of Malaysia (MOHE) -Fundamental Research Grant } \\
\text { Scheme } 2013\end{array}$ & FRGS13-079-0320 \\
\hline TWAS-COMSTECH Research Grant & REF: $15-333$ RG/REN/AS_C- \\
\hline
\end{tabular}

View funding text

Publisher

PERGAMON-ELSEVIER SCIENCE LTD, THE BOULEVARD, LANGFORD LANE, KIDLINGTON, OXFORD OX5 1GB, ENGLAND

Categories / Classification

Research Areas: Engineering; Environmental Sciences \& Ecology

Web of Science Categories: Engineering, Environmental; Environmental Sciences

See more data fields 


\section{Cited References: 48}

1. Optical, structural and catalytic evaluation of gamma-irradiation synthesized Ag/PVA nanocomposite films

By: Ali, Z.I.; Saleh, H.H.; Afify, T.A.

Int. J. Eng. Res. Technol. Volume: 3 Pages: 1527-1538 Published: 2014

URL: https://doi.org/10.1017/CB09781107415324.004

2. A comprehensive review on biodiesel as an alternative energy resource and its characteristics

Times Cited: 556

By: Atabani, A. E.; Silitonga, A. S.; Badruddin, Irfan Anjum; et al.

RENEWABLE \& SUSTAINABLE ENERGY REVIEWS Volume: 16 Issue: 4 Pages: 2070-2093 Published: MAY 2012

3. Removal of residual palm oil-based biodiesel catalyst using membrane ultra-filtration technique: An optimization study

Times Cited: 9

By: Atadashi, I. M.; Aroua, M. K.; Aziz, A. R. Abdul; et al.

ALEXANDRIA ENGINEERING JOURNAL Volume: 53 Issue: 3 Pages: 705-715 Published: SEP 2014

4. Preparation of calcium-phosphate bioceramics from natural resources

Times Cited: 83

By: Balazsi, Csaba; Weber, Ferenc; Kover, Zsuzsanna; et al.

JOURNAL OF THE EUROPEAN CERAMIC SOCIETY Volume: 27 Issue: 2-3 Pages: 1601-1606 Published: 2007

5. Biodiesel-quality, emissions and by-products

Times Cited: 2

By: Barabas, I.; Todorut, I.-A.

Biodiesel: Quality, Emissions and by-Products Pages: 392 Published: 2011

URL: http://doi.org/10.5772/2284

6. Biodiesel Science and Technology: From Soil to Oil

Times Cited: 50

By: Bart, JCJ; Palmeri, N; Cavallaro, S

BIODIESEL SCIENCE AND TECHNOLOGY: FROM SOIL TO OIL Book Series: Woodhead Publishing Series in Energy Issue: 7 Pages: 1-840

Published: 2010

Publisher: WOODHEAD PUBL LTD, ABINGTON HALL ABINGTON, CAMBRIDGE CB1 6AH, CAMBS, ENGLAND

7. Non-conventional seed oils as potential feedstocks for future biodiesel industries: a brief review.

Times Cited: 5

By: Basumatary Sanjay

Research Journal of Chemical Sciences Volume: 3 Issue: 5 Pages: 99-103 Published: 2013

8. Enzymatic transesterification of soybean oil with ethanol using lipases immobilized on highly crystalline PVA microspheres

Times Cited: 8

By: Bergamasco, Juliana; de Araujo, Marcelo V.; de Vasconcellos, Adriano; et al.

BIOMASS \& BIOENERGY Volume: 59 Pages: 218-233 Published: DEC 2013

9. Kinetics studies of synthesis of biodiesel from waste frying oil using a heterogeneous catalyst derived from snail shell

By: Birla, Ashish; Singh, Bhaskar; Upadhyay, S. N.; et al.

BIORESOURCE TECHNOLOGY Volume: 106 Pages: 95-100 Published: FEB 2012

10. Performance of calcium oxide as a heterogeneous catalyst in biodiesel production: A review

Times Cited: 127

By: Boey, Peng-Lim; Maniam, Gaanty Pragas; Abd Hamid, Shafida

CHEMICAL ENGINEERING JOURNAL Volume: 168 Issue: 1 Pages: 15-22 Published: MAR 152011

11. Application of eggshell wastes as a heterogeneous catalyst for biodiesel production

By: Buasri, A.

Sustainable Energy Volume: 1 Issue: 2 Pages: 7-13 Published: 2013

12. Development of cellulose nanofibres from coconut husk

Times Cited: 2

By: Chandrahasa, R.; Rajamane, N.P.

Int. J. Emerg. Technol. Adv. Eng. Volume: 4 Pages: 88-93 Published: 2014

Times Cited: 10

13. Characterization of calcium oxide catalysts from natural sources and their applicatio
By: Correia, Leandro Marques; Alves Saboya, Rosana Maria; Campelo, Natalia de Sousa; et al. 
14. Performance evaluation of biodiesel from used domestic waste oils: A review

Times Cited: 49

By: Diya'uddeen, Basheer Hasan; Aziz, A. R. Abdul; Daud, W. M. A. W.; et al.

PROCESS SAFETY AND ENVIRONMENTAL PROTECTION Volume: 90 Issue: 3 Special Issue: SI Pages: 164-179 Published: MAY 2012

15. In-situ transesterification of rapeseed and cost indicators for biodiesel production

Times Cited: 23

By: El-Enin, S. A. Abo; Attia, N. K.; El-lbiari, N. N.; et al.

RENEWABLE \& SUSTAINABLE ENERGY REVIEWS Volume: 18 Pages: 471-477 Published: FEB 2013

16. Statistical optimization of biodiesel production from sunflower waste cooking oil using basic heterogeneous biocatalyst prepared from eggshells

By: El-Gendy, N.S.; Deriase, S.F.; Hamdy, A.; et al.

Egyptian Journal of Petroleum Volume: 24 Issue: 1 Pages: 37-48 Published: March 2015

17. Temperature effects on egg shells investigated by XRD, IR and ESR techniques

Times Cited: 40

By: Engin, B; Demirtas, H; Eken, M

RADIATION PHYSICS AND CHEMISTRY Volume: 75 Issue: 2 Pages: 268-277 Published: FEB 2006

18. Production of biodiesel from non-edible oil and its properties

Times Cited: 9

By: Gashaw, A; lakachew, A

Int J Sci Environ Technol Volume: 3 Pages: 1544-1562 Published: 2014

19. Comparison of delignified coconuts waste and cactus for fuel-ethanol production by the simultaneous and semi-simultaneous saccharification and fermentation strategies

By: Goncalves, Fabiano Avelino; Ruiz, Hector A.; Nogueira, Cleitiane da Costa; et al.

FUEL Volume: 131 Pages: 66-76 Published: SEP 12014

20. Title: [not available]

Times Cited: 2

By: Hariharan, M.; Varghese, N.; Cherian, A.B.; et al.

Synthesis and characterisation of $\mathrm{CaCO} 3$ (Calcite) nano particles from cockle shells using chitosan as precursor Published: 2014

[Show additional data]

21. Transesterification of used cooking oil over alkali metal ( $\mathrm{Li}, \mathrm{Na}, \mathrm{K}$ ) supported rice husk silica as potential solid base catalyst By: Hindryawati, Noor; Maniam, Gaanty Pragas; Karim, Md. Rezaul; et al.

ENGINEERING SCIENCE AND TECHNOLOGY-AN INTERNATIONAL JOURNAL-JESTECH Volume: 17 Issue: 2 Pages: 95-103 Published: JUN 2014

22. Nano-magnetic catalyst $\mathrm{KF} / \mathrm{CaO}-\mathrm{Fe} 304$ for biodiesel production

Times Cited: 90

By: Hu, Shengyang; Guan, Yanping; Wang, Yun; et al.

APPLIED ENERGY Volume: 88 Issue: 8 Pages: 2685-2690 Published:AUG 2011

23. Egg shell as eco - friendly catalyst for transesterification of rapeseed oil: optimization for biodiesel production

Times Cited: 1

By: Jazie, A.A.; Pramanik, H.; Sinha, A.S.K.

Int. J. Sustain. Dev. Green Econ. Volume: 2 Pages: 2315-4721 Published: 2013

Spec. Issue

24. Egg shell waste-catalyzed transesterification of mustard oil: optimization using response surface methodology (RSM)

Times Cited: 1

By: Jazie, A.A.; Pramanik, H.; Sinha, A.S.K.

20122 INT C POW EN Volume: 56 Pages: 52-57 Published: 2012

URL: http://doi.org/10.7763/1PCSIT.2012.V56.10

25. Transesterification of peanut and rapeseed oils using waste of animal bone as cost effective catalyst

Times Cited: 3

By: Jazie, Ali A.; Pramanik, H.; Sinha, A. S. K.

MATERIALS FOR RENEWABLE AND SUSTAINABLE ENERGY Volume: 2 Issue: 2 Article Number: 11 Published: JUN 2013

26. Industrial eggshell wastes as the heterogeneous catalysts for microwave-assisted biodiesel production

Times Cited: 63

By: Khemthong, P.; Luadthong, C.; Nualpaeng, W.; et al.

CATALYSIS TODAY Volume: 190 Issue: 1 Pages: 112-116 Published: AUG 12012

27. Optimization and kinetics of sunflower oil methanolysis catalyzed by calcium oxide-based catalyst derived from palm kernel 
shell biochar

By: Kostic, Milan D.; Bazargan, Alireza; Stamenkovic, Olivera S.; et al.

FUEL Volume: 163 Pages: 304-313 Published: JAN 12016

28. Transesterification of triacylglycerols over calcium oxide as heterogeneous catalyst

Times Cited: 11

By: Lengyel, J.; Cvengrosova, Z.; Cvengros, J.

Petroleum Coal Volume: 51 Pages: 216-224 Published: 2009

29. Kinetic comparison of two basic heterogenous catalysts obtained from sustainable resources for transesterification of waste

Times Cited: 17 cooking oil

By: Moradi, G. R.; Mohadesi, M.; Ghanbari, M.; et al.

BIOFUEL RESEARCH JOURNAL-BRJ Volume: 2 Issue: 2 Pages: 236-241 Published: SPR 2015

30. Transesterification of soybean oil using combusted oyster shell waste as a catalyst

Times Cited: 160

By: Nakatani, Nobutake; Takamori, Hitoshi; Takeda, Kazuhiko; et al.

BIORESOURCE TECHNOLOGY Volume: 100 Issue: 3 Pages: 1510-1513 Published: FEB 2009

Showing $\mathbf{3 0}$ of $48 \quad$ View All in Cited References page

\section{Clarivate}

Accelerating innovation (c) 2019 Clarivate Copyright notice Terms of use Privacystatement Cookie policy

Sign up for the Web of Science newsletter Follow us 